\title{
Prediction of quantization of magnetic flux in double-layer exciton superfluids
}

Rademaker, L.; Zaanen, J.; Hilgenkamp, J.W.M.

\section{Citation}

Rademaker, L., Zaanen, J., \& Hilgenkamp, J. W. M. (2011). Prediction of quantization of magnetic flux in double-layer exciton superfluids. Physical Review B, 83(1), 012504. doi:10.1103/PhysRevB.83.012504

Version: $\quad$ Not Applicable (or Unknown)

License: $\quad$ Leiden University Non-exclusive license

Downloaded from: https://hdl.handle.net/1887/61283

Note: To cite this publication please use the final published version (if applicable). 


\title{
Prediction of quantization of magnetic flux in double-layer exciton superfluids
}

\author{
Louk Rademaker, ${ }^{1, *}$ Jan Zaanen, ${ }^{1}$ and Hans Hilgenkamp ${ }^{1,2}$ \\ ${ }^{1}$ Institute-Lorentz for Theoretical Physics, Leiden University, P.O. Box 9506, NL-2300 RA Leiden, The Netherlands \\ ${ }^{2}$ Faculty of Science and Technology and MESA + Institute for Nanotechnology, University of Twente, \\ P.O. Box 217, NL-7500 AE Enschede, The Netherlands
}

(Received 8 December 2010; published 24 January 2011)

\begin{abstract}
Currently, there is no way to detect unambiguously the possible phase coherence of an exciton condensate in an electron-hole double layer. Here, we show that, despite the fact that excitons are charge neutral, the double-layer exciton superfluid exhibits a diamagnetic response. In devices with specific circular geometry, the magnetic-flux threading between the layers must be quantized in units of $\frac{h}{e} \chi_{m}$, where $\chi_{m}$ is the diamagnetic susceptibility of the device. We discuss possible experimental realizations of the predicted unconventional flux quantization.
\end{abstract}

DOI: 10.1103/PhysRevB.83.012504

PACS number(s): 05.30.-d, 71.35.Lk, 73.20.Mf

\section{INTRODUCTION}

It is well known that a quantization condition applies to the magnetic flux enclosed by a superconducting cylinder. ${ }^{1,2}$ This effect is due to the coherence inherent to the superfluid phase, causing quantum-mechanical principles to become manifest in macroscopic objects. ${ }^{3}$ Such a superfluid phase is also predicted for excitons in spatially separated electron and hole layers. ${ }^{4-6}$ Excitons are bound pairs of electrons and holes, and they have a long history as optical excitations in semiconductors and insulators. Recent technological developments allowed for the realization of devices ${ }^{7,8}$ that consist of a pair of two-dimensional layers, where the electrons are confined to one layer and the holes to the other layer as shown in Fig. 1. When the interlayer distance is small enough (typically of the order of $10 \mathrm{~nm}$ ), the interlayer Coulomb interaction becomes strong enough to bind the electrons and the holes in interlayer excitons. An insulating barrier separates the layers to prevent annihilation of the excitons by tunneling. The layers themselves can be composed of semiconductor quantum wells, ${ }^{7}$ graphene sheets, ${ }^{9-13}$ complex oxides, ${ }^{14,15}$ or even topological insulators. ${ }^{16}$

Excitons are bosons and at finite densities, they should eventually form a Bose-Einstein condensate at sufficiently low temperatures. There are indications in several experiments ${ }^{7,8}$ that exciton condensates were formed, but there is no way to detect unambiguously the onset of macroscopic superfluid coherence in these double-layer exciton systems. ${ }^{17,18}$ Here, we predict an unconventional magnetic-flux quantization effect to occur in double-layer exciton superfluids, as shown in Fig. 2, and discuss designs for a device to measure this universal electromagnetic signature of the exciton BoseEinstein condensate.

\section{GINZBURG-LANDAU THEORY}

Let us consider the Ginzburg-Landau order-parameter theory for a double-layer system. Since the direction of the electric dipole is fixed in the double-layer geometry, the exciton superfluid is characterized by just a complex scalar orderparameter field $\Psi(\vec{x}) \equiv|\Psi(\vec{x})| e^{i \phi(\vec{x})}$ along a two-dimensional (2D) surface, the square of which gives the superfluid density $\rho(\vec{x})=|\Psi(\vec{x})|^{2}$. For a charged superfluid (superconductor) with boson charge $q$, electromagnetism is incorporated by replacing ordinary derivatives with covariant derivatives $\vec{D}$,

$$
\hbar \vec{D}=\hbar \vec{\nabla}+i q \vec{A}(\vec{x}),
$$

where $\vec{A}(\vec{x})$ is the vector potential. In the charge-neutral exciton superfluid, the electron and hole constituents of an exciton form an electric dipole $e \vec{d}$ and, consequently, the covariant derivative associated with exciton matter must equal $^{19}$

$$
\hbar \vec{D}=\hbar \vec{\nabla}+i e[\vec{A}(\vec{x}+\vec{d} / 2)-\vec{A} \vec{x}-\vec{d} / 2)],
$$

where the electron is positioned at $\vec{x}-\vec{d} / 2$ and the hole at $\vec{x}+\vec{d} / 2$. For small interlayer distance $\vec{d}$, the vector potential can be expanded in a Taylor series. In addition, since the vector potential $\vec{A}$ along the $2 \mathrm{D}$ superfluid surface is only sourced by in-plane currents, we can impose that the gradient of the vector-potential component perpendicular to the surface is zero, i.e.,

$$
\left.\vec{\nabla}^{\prime}\left(\vec{d} \cdot \vec{A}\left(\vec{x}^{\prime}\right)\right)\right|_{\vec{x}^{\prime}=\vec{x}}=0 .
$$

This implies that the above vector-potential difference can be written completely in terms of the real magnetic field

$$
\begin{aligned}
& \vec{A}(\vec{x}+\vec{d} / 2)-\vec{A}(\vec{x}-\vec{d} / 2) \\
& \quad=-\vec{d} \times\left.\sum_{k=0}^{\infty} \frac{1}{(2 k+1) !}\left(\frac{\vec{d}}{2} \cdot \vec{\nabla}^{\prime}\right)^{2 k} \vec{B}\left(\vec{x}^{\prime}\right)\right|_{\vec{x}^{\prime}=\vec{x}} .
\end{aligned}
$$

Up to first order, the exciton covariant derivative turns into

$$
\hbar \vec{D}=\hbar \vec{\nabla}-i e \vec{d} \times \vec{B} .
$$

This is an interesting structure viewed from a theoretical perspective. Equation (5) corresponds to the covariant derivatives of a SU(2) gauge theory with gauge fields $A_{i}^{a}=\epsilon^{i a k} B_{k}$. Here, the SU(2) gauge fields are actually physical fields fixed by Maxwell's equations. Using these considerations, we can write down a general Ginzburg-Landau free energy as

$$
\begin{aligned}
F[\Psi]= & \int d^{2} x\left[\alpha|\Psi|^{2}+\frac{1}{2} \beta|\Psi|^{4}+\frac{\hbar^{2}}{2 m^{*}}(\nabla|\Psi|)^{2}\right. \\
& \left.+\frac{1}{2 m^{*}}(\hbar \vec{\nabla} \phi-e \vec{d} \times \vec{B})^{2}|\Psi|^{2}+d \frac{B^{2}}{2 \mu_{0}}\right] .
\end{aligned}
$$




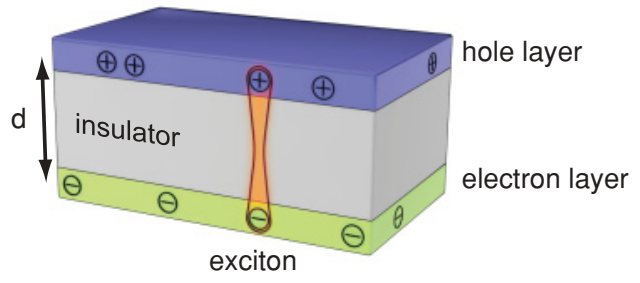

FIG. 1. (Color online) Excitons in double-layer devices. Doublelayer devices consist of an electron layer (in green) parallel to a hole layer (blue), separated by an insulating barrier (gray). Electronhole attraction leads to the formation of excitons. At sufficiently low temperatures, the excitons can form a Bose-Einstein condensate.

The parameters $\alpha$ and $\beta$ can be written formally as a function of the superfluid density and the critical magnetic field $B_{c}$. Minimization of the free energy, assuming a constant order parameter, yields

$$
\begin{gathered}
\alpha=-d \frac{B_{c}^{2}}{\mu \rho}, \\
\beta=-\frac{\alpha}{\rho} .
\end{gathered}
$$

\section{ELECTROMAGNETIC RESPONSE}

The direct coupling to physical fields changes the rules drastically as compared to normal superconductors. We define the exciton supercurrent as the standard Noether current ${ }^{3} \vec{j} \equiv$ $\frac{\hbar \rho}{m^{*}} \vec{\nabla} \phi$. Consequently, minimizing the free energy for a fixed applied magnetic field $\vec{B}$ perpendicular to the dipole moment yields the exciton supercurrent response

$$
\vec{j} \equiv \frac{\hbar \rho}{m^{*}} \vec{\nabla} \phi=\frac{\rho e}{m^{*}} \vec{d} \times \vec{B} .
$$

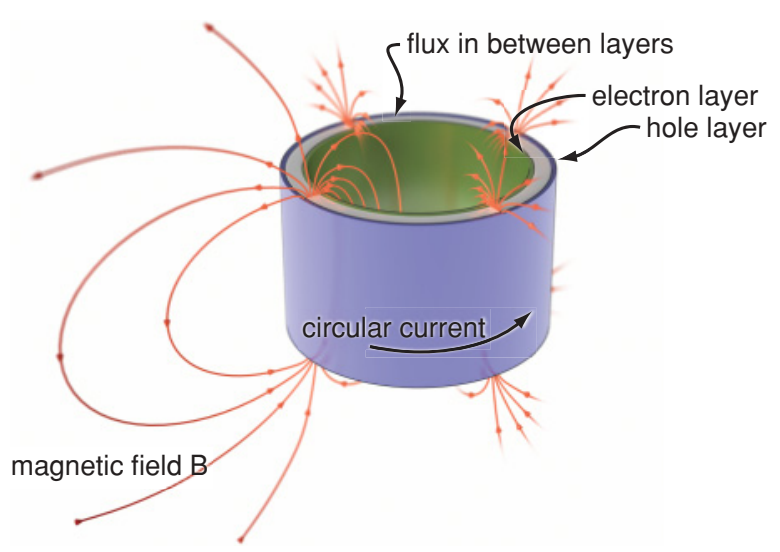

FIG. 2. (Color online) Flux trapping in a cylindrical exciton superfluid. The proposed device consists of a concentric ring structure of radius $r$, composed of an electron layer (green) and hole layer (blue). Due to the macroscopic coherence of the exciton superfluid, the angular current must be quantized. By application of an external axial magnetic field, one can induce some number of current quanta. In the absence of the external field, the current quanta remain, which induces a magnetic field as shown (red lines). The trapped magnetic flux in-between the layers must be quantized according to $\Phi=\frac{h}{e} \chi_{m} n$, where $\chi_{m}$ is defined in Eq. (11).
This result is closely related to spin superfluids, ${ }^{20}$ where a physical field $\mathrm{SU}(2)$ structure arises through spin-orbit coupling. ${ }^{21}$ The analog of Eq. (9) is the spin Hall equation ${ }^{22}$ $j_{j}^{i}=\sigma_{\mathrm{s}} \varepsilon^{i j k} E_{k} \rightarrow \vec{j}=-\sigma \vec{d}_{m} \times \vec{E}$. We conclude that the spin superfluid formed from magnetic dipoles is the electromagnetic dual of the exciton (electric dipole) superfluid.

In the double-layer system, the electric charges forming the exciton dipoles are confined in the separate layers. Hence, the exciton supercurrent can be decomposed into the separated electron and hole surface currents. According to Ampère's law, a surface current induces a discontinuity in the magnetic-field components parallel to the surface,

$$
\Delta \vec{B}(\vec{x})=\mu_{0} \vec{K}(\vec{x}) \times \hat{n},
$$

where $\hat{n}$ is the normal vector to the surface and $\vec{K}(\vec{x})$ is an electric surface current density. Consequently, an exciton supercurrent reduces the magnetic field in-between the electron and hole layers. The double layer therefore acts as a (nonperfect) diamagnet with magnetic susceptibility

$$
\chi_{m}=-\frac{e^{2} \rho d \mu_{0}}{m^{*}} .
$$

For typical parameters, $\rho=0.4 \mathrm{~nm}^{-2}, d=20 \mathrm{~nm}$, and $m^{*}=2 m_{e}$; the magnetic susceptibility equals $\chi_{m}=-10^{-4}$, comparable to what is found in diamagnets such as gold or diamond. In semiconductor quantum wells, the exciton mass is smaller than the free electron mass $m_{e}$, which enhances the diamagnetic susceptibility even further. ${ }^{?}$

\section{FLUX QUANTIZATION EFFECTS}

Imposing single valuedness on the order parameter implies that, for any given contour $C$ inside a superfluid, $\oint_{C} \vec{\nabla} \phi \cdot \vec{d} l=$ $\oint_{C} \vec{j} \cdot \vec{d} l=2 \pi n$, where $n$ is an integer. Therefore, circular supercurrents must be quantized, which can be seen by topological defects in the dipolar superfluid. ${ }^{20,23,24}$ In general, metastability of superflows requires a nontrivial topology of the superfluid. ${ }^{3}$ Unlike in other superfluids, the SU(2) structure of dipolar superfluids implies the possibility of more complicated topologies, which can not be obtained by creating defects in the superfluid.

Consider a cylindrical device of radius $r$ consisting of two concentric layers, as shown in Fig. 2, with the electric dipole moment $\vec{d}$ of the excitons pointing in the radial direction. For this geometry, the current-dependent term in the free energy can be written as

$$
F[\Psi] \sim \oint d \theta\left(\frac{h}{e} \partial_{\theta} \phi-B_{z} 2 \pi r d\right)^{2},
$$

where $\oint_{C} d \theta \partial_{\theta} \phi=2 \pi n$ with $n$ integer valued and $B_{z}$ the external magnetic field. Note that the flux going in-between the two layers equals, up to first order, $\Phi=B_{z} 2 \pi r d$. Minimization of Eq. (12) shows that current quanta can be induced by an axial magnetic field. In the absence of the external field, the current $\vec{j} \sim n$ induces a magnetic flux in-between the layers, according to Ampère's law (10), with a magnitude

$$
\Phi=\frac{h}{e} \chi_{m} n \equiv \Phi_{0} \chi_{m} n
$$


This is our central result: In the cylindrical double-layer geometry, the magnetic flux going in-between the sample layers must be quantized in units of $\chi_{m}$ times the fundamental flux quantum $\Phi_{0}=\frac{h}{e}$. Notice that this flux quantization effect is quite different from the one realized in superconductors. In the double-layer exciton condensate, the supercurrent is induced by the magnetic field $\vec{B}$ rather than the gauge field $\vec{A}$ as in the London equation, while the quantized amount of flux equals $d \oint \vec{B} \cdot \vec{d} l$ instead of $\oint \vec{A} \cdot \vec{d} l=\iint_{\Sigma} \vec{B}$. $\overrightarrow{d \Sigma}$ for superconductors. In combination, these two basic differences add up to a universal expression for the flux quantization $\Phi=\frac{h}{e^{*}} \chi_{m} n$ that applies to both superconductors and exciton condensates, where $e^{*}=-2 e$ and $\chi_{m}=-1$ for superconductors.

\section{PHASE SLIP AND PHASE PINNING}

Is the strength of the condensate sufficient to trap the flux? When the external field is switched off, the flux carrying state is metastable and the system can return to the ground state by locally destroying the condensate. The condensate can only be destroyed over lengths greater than the Ginzburg-Landau coherence length

$$
\xi=\frac{\hbar}{\sqrt{\left|2 m^{*} \alpha\right|}}
$$

and, consequently, the energy required to break the condensate over a region $\xi$ wide along a cylinder of length $z$ is

$$
\delta F_{b}=\frac{1}{2} \hbar z\left(\frac{d \rho}{2 m^{*} \mu}\right)^{1 / 2} B_{c} .
$$

Locally destroying the condensate is only favorable if this energy is lower than the energy stored in the magnetic field, which is $\delta F_{m}=\frac{B^{2}}{2 \mu} 2 \pi r d$. We conclude that a phase slip will not occur as long as the trapped magnetic flux $\Phi=\Phi_{0} \chi_{m} n$ stays below a threshold value

$$
\Phi^{2}<\left(\frac{\left|\chi_{m}\right|}{2}\right)^{1 / 2} \Phi_{0} B_{c} r d,
$$

where $B_{c}$ is the critical magnetic field. With the typical parameters stated above and $r=100 \mu \mathrm{m}$, the critical field must exceed $5 \mathrm{nT}$ to trap one flux quantum. Since the critical magnetic field of bilayer superfluids is proposed to lie in the orders of tens of Tesla, ${ }^{19}$ a phase slip is improbable.

Another possible complication is that annihilation of excitons by tunneling causes the phase to be pinned, which introduces a threshold for the formation of stable currents. Microscopic tunneling can be incorporated via an extra term in the Ginzburg-Landau free energy

$$
F_{t}=-2 t \int d^{2} x \frac{|\Psi|}{L} \cos \phi,
$$

where $L$ is the in-plane lattice constant and $t$ is a microscopic tunneling energy. This phase pinning lowers the energy of the state where no flux is trapped, which introduces a threshold for the trapping of magnetic-flux quanta. It is only possible to trap $n$ magnetic-flux quanta if the microscopic tunneling energy $t$ satisfies

$$
2 t<n^{2} \frac{\hbar^{2}}{2 m^{*} r^{2}} \sqrt{\rho} L .
$$

This corresponds, given the typical parameters mentioned above, to $t<0.3 \mathrm{peV}$ (picoelectronvolt) for the first flux quantum.

In order to estimate a value for $t$, let us imagine that the device is fabricated from copper-oxide layers. The hopping energy in cuprates between two adjacent $\mathrm{CuO}_{2}$ layers ranges from approximately $10^{-1} \mathrm{eV}$ for LSCO compounds to $10^{-3} \mathrm{eV}$ for Bi-based compounds..$^{25,26}$ Let us now assume that the hopping energy between more distant $\mathrm{CuO}_{2}$ layers falls off exponentially. A distance $d=20 \mathrm{~nm}$ between the hole and electron layers corresponds roughly to $30 \mathrm{CuO}_{2}$ layers, so that the tunneling energy equals $t \approx e^{-30} 10^{-3}=10^{-16} \mathrm{eV}$. This estimate lies well below the maximum value of $t$ obtained in Eq. (18). However, the precise value of $t$ is highly sample specific and needs to be checked for each separate sample.

\section{EXPERIMENTAL REALIZATION}

The experimental protocol to test the flux quantization is as follows: Apply an axial magnetic field of magnitude $B_{\text {ext }}$ above the critical temperature $T_{c}$, and cool the device below $T_{c}$ such that a circular current quantum is frozen in. The magnitude of the current is determined by the strength of the applied flux: if $\Phi_{\text {ext }}<\frac{1}{2} \Phi_{0}$, no current is induced; for $\frac{1}{2} \Phi_{0}<\Phi_{\text {ext }}<\frac{3}{2} \Phi_{0}$, one current quantum is induced, etc. The magnetic field corresponding to $\frac{1}{2} \Phi_{0}$ is typically $B_{\text {ext }}=0.2 \mathrm{mT}$. Upon removing the external magnetic field, a trapped flux equal to $\Phi_{0} \chi_{m} n$ remains, corresponding to a field strength of $50 \mathrm{nT}$. These numbers do not pose a problem of principle for the experimental realization of such a flux trapping device.

Based on existing technology, one can envision various practical realizations of the concentric $p-n$ doped ring geometry, while it is anticipated that further technology developments will create additional opportunities. Using $p$ - and $n$-doped complex oxide compounds, such as cuprate perovskites, multilayer thin film structures can be fabricated in the desired ring geometry. Using the proven edge-junction technology, ${ }^{27,28}$ the structure sketched in Fig. 3 can readily be fabricated by, e.g., pulsed laser deposition and Ar-ion beam etching. As a barrier layer, $\mathrm{SrTiO}_{3}$ can be used, with a typical thickness of 10-100 nm, or another insulating oxide that grows epitaxially on top of the etched base electrode. To

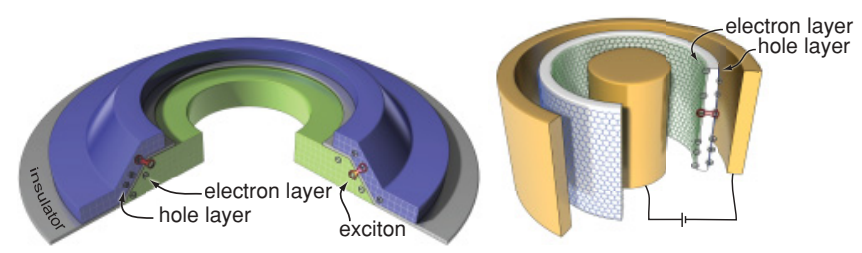

FIG. 3. (Color online) Schematic representations of possible practical realizations of the concentric ring geometry comprised of $p$ - and $n$-doped layers. Left: Using epitaxially grown complex oxide thin films. Right: Using doubly gated graphene double layers. 
guarantee an epitaxial growth of all the layers, the angle $\alpha$ is best kept below about $25^{\circ}$, but this does not fundamentally alter the physics of the flux quantization as presented in this brief report.

A second possible practical realization is based on doubleside gated, double-layer graphene. Recently, the growth of large-area graphene films has been demonstrated on $\mathrm{Cu}$ foils, using a high-temperature chemical vapor deposition process. ${ }^{29}$ Interestingly, a continuous growth was achieved over grain boundaries and surface steps. From this, it feasible to expect that one can also grow a closed graphene tube around a copper cylinder, which would basically be a carbon nanotube with predetermined radius. Covering this with an appropriate epitaxial barrier layer, e.g., $10 \mathrm{~nm}$ of $\mathrm{Al}_{2} \mathrm{O}_{3}$ and a second graphene sheet, which may also be grown by physical or chemical vapor deposition techniques, would then result in the wanted concentric cylinder configuration. Subsequently, the copper can be etched away and the concentric cylinder can be transferred to an appropriate carrier, which can even be made out of plastic. ${ }^{30}$ This would straightforwardly allow the realization of a doubly gated configuration, as depicted in Fig. 3.

\section{CONCLUSION}

We have shown that dipolar exciton condensates exhibit a different form of magnetic flux quantization. Whereas the values for the magnetic flux quanta are reduced by a factor $2 \chi_{m} \approx 10^{-4}-10^{-3}$ compared to the standard flux quanta in superconducting rings, it is anticipated that the flux quantization is measurable using scanning SQUID microscopy. This would provide an unambiguous test for the macroscopic phase coherence associated with an exciton Bose-Einstein condensate.

\section{ACKNOWLEDGMENTS}

We thank A. V. Balatsky, P. H. Kes, J. van der Brink, and P. B. Littlewood for helpful discussions, and J. Huijben for designing the figures. This work was supported by the Netherlands Organization for Scientic Research (NWO). *rademaker@lorentz.leidenuniv.nl

${ }^{1}$ B. S. Deaver and W. M. Fairbank, Phys. Rev. Lett. 7, 43 (1961).

${ }^{2}$ R. Doll and M. Näbauer, Phys. Rev. Lett. 7, 51 (1961).

${ }^{3}$ A. J. Leggett, Quantum Liquids: Bose Condensation and Cooper Pairing in Condensed-Matter Physics (Oxford University Press, Oxford, 2006)

${ }^{4}$ S. I. Shevchenko, Fiz. Nizk. Temp. 2, 505 (1976) [Sov. J. Low Temp. Phys. 2, 251 (1976)].

${ }^{5}$ Yu. E. Lozovik and V. I. Yudson, Zh. Eksp. Teor. Fiz. 71, 738 (1976) [Sov. Phys. JETP 44, 389 (1976)].

${ }^{6} \mathrm{~S}$. A. Moskalenko and D. W. Snoke, Bose-Einstein Condensation of Excitons and Biexcitons and Coherent Nonlinear Optics with Excitons (Cambridge University Press, Cambridge, 2000).

${ }^{7}$ L. V. Butov, Solid State Commun. 127, 89 (2003).

${ }^{8}$ J. P. Eisenstein and A. H. MacDonald, Nature (London) 432, 691 (2004)

${ }^{9}$ Yu. E. Lozovik and A. A. Sokolik, Pis'ma Zh. Eksp. Teor. Fiz. 87, 61 (2008) [JETP Lett. 87, 55 (2008)].

${ }^{10}$ C.-H. Zhang and Y. N. Joglekar, Phys. Rev. B 77, 233405 (2008).

${ }^{11}$ R. Dillenschneider and J. H. Han, Phys. Rev. B 78, 045401 (2008).

${ }^{12}$ H. Min, R. Bistritzer, J.-J. Su, and A. H. MacDonald, Phys. Rev. B 78, 121401(R) (2008).

${ }^{13}$ M. Yu. Kharitonov and K. B. Efetov, Phys. Rev. B 78, 241401 (2008).

${ }^{14}$ R. Pentcheva et al., Phys. Rev. Lett. 104, 166804 (2010).
${ }^{15}$ A. J. Millis and D. G. Schlom, Phys. Rev. B 82, 073101 (2010).

${ }^{16}$ B. Seradjeh, J. E. Moore, and M. Franz, Phys. Rev. Lett. 103, 066402 (2009).

${ }^{17}$ D. W. Snoke, Phys. Status Solidi B 238, 389 (2003); Adv. in Cond. Mat. Phys. 2011, 938609 (2010).

${ }^{18}$ J.-J. Su and A. H. MacDonald, Nat. Phys. 4, 799 (2008).

${ }^{19}$ A. V. Balatsky, Y. N. Joglekar, and P. B. Littlewood, Phys. Rev. Lett. 93, 266801 (2004).

${ }^{20}$ B. W. A. Leurs, Z. Nazario, D. I. Santiago, and J. Zaanen, Ann. Phys. (NY) 323, 907 (2008).

${ }^{21}$ A. S. Goldhaber, Phys. Rev. Lett. 62, 482 (1989).

${ }^{22}$ S. Murakami, N. Nagaosa, and S.-C. Zhang, Science 301, 1348 (2003).

${ }^{23}$ E. Babaev, Phys. Rev. B 77, 054512 (2008).

${ }^{24}$ B. Seradjeh, H. Weber, and M. Franz, Phys. Rev. Lett. 101, 246404 (2008).

${ }^{25}$ S. L. Cooper and K. E. Gray, in Physical Properties of High Temperature Superconductors IV, edited by D. M. Ginsberg (World Scientific, Singapore, 1994).

${ }^{26}$ D. G. Clarke and S. P. Strong, Adv. Phys. 46, 545 (1997)

${ }^{27}$ J. Gao, W. A. M. Aarnink, G. J. Gerritsma, and H. Rogalla, Phys. C (Amsterdam) 171, 126 (1990).

${ }^{28} \mathrm{H}$. Hilgenkamp et al., Nature (London) 422, 50 (2003).

${ }^{29} \mathrm{X}$. Li et al., Science 324, 1312 (2009).

${ }^{30}$ S. Bae et al., Nat. Nanotech. 5, 574 (2010). 